\title{
RANK-FINITENESS FOR G-CROSSED BRAIDED FUSION CATEGORIES
}

\author{
COREY JONES, SCOTT MORRISON, DMITRI NIKSHYCH, ERIC C. ROWELL
}

\begin{abstract}
We establish rank-finiteness for the class of $G$-crossed braided fusion categories, generalizing the recent result for modular categories and including the important case of braided fusion categories. This necessitates a study of slightly degenerate braided fusion categories and their centers, which are interesting for their own sake.
\end{abstract}

\section{INTRODUCTION}

The question of whether there are finitely many fusion categories with a fixed number of isomorphism classes of simple objects (i.e., fixed rank) was first raised by Ostrik in O1, where an affirmative answer was given for rank 2. In ENO1] the special case of categories with integral Frobenius-Perron dimension (i.e. weakly integral categories) was also settled. Around 2003 Wang conjectured that there are always finitely many modular categories of a given fixed rank, which was explicitly verified for rank at most 4 . A proof of this rank-finiteness conjecture was obtained recently BNRW]. The main goal of this article is to extend rank-finiteness to the generality of $G$-crossed braided fusion categories, which includes the important case of braided fusion categories, and does not require the existence of a spherical structure.

The primary obstacle to overcome is the existence of slightly degenerate braided fusion categories, with symmetric center equivalent the braided fusion category sVec of super vector spaces. These are interesting in their own right, with the main open question being whether or not every slightly degenerate braided fusion category admits a minimal non-degenerate extension. As a step towards answering this question we analyze the structure of the Drinfeld center of a slightly degenerate braided fusion category.

As a technical tool, we prove a bound on the rank of invertible $(\mathcal{C}-\mathcal{D})$-bimodule categories. In particular, we show that for any invertible $\mathcal{C}$-bimodule category, $\operatorname{rank}(\mathcal{M}) \leq \operatorname{rank}(\mathcal{C})$. In addition, we show that the set of equivalence classes of invertible bimodule categories realizing this bound forms a subgroup of $\operatorname{BrPic}(\mathcal{C})$, and discuss some examples.

Date: February 19, 2019.

ECR is partially supported by NSF grant DMS-1664359. DN was partially supported by the NSA grant H98230-16-1-0008 and the NSF grant DMS-1801198. This paper was initiated while ECR and DN were visiting CJ and SM at the Australian National University, and gratefully acknowledge the support of that institution. 


\section{Preliminaries}

We work over an algebraically closed field $k$ of characteristic 0 . All fusion categories and their module categories are assumed to be $k$-linear. For the basics of the theory of fusion categories we refer the reader to [EGNO] and [DGNO].

By the rank of a fusion category we mean the number of isomorphism classes of its simple objects.

Let Vec and sVec denote the braided fusion categories of vector spaces and super vector spaces over $k$. For any braided fusion category $\mathcal{C}$ let $\mathcal{Z}_{\text {sym }}(\mathcal{C})$ denote its symmetric (or Müger) center.

Definition 2.1. A braided fusion category $\mathcal{C}$ is called slightly degenerate DNO] if $\mathcal{Z}_{\text {sym }}(\mathcal{C})=$ sVec. A slightly degenerate ribbon fusion category is called supermodular.

The smallest example of a slightly degenerate braided fusion category is $\mathrm{sVec}$ itself.

Example 2.2. One can construct a slightly degenerate braided fusion category as follows. Let $\tilde{\mathcal{C}}$ be a non-degenerate braided fusion category and let $\mathrm{sVec} \hookrightarrow \tilde{\mathcal{C}}$ be a braided tensor functor (it is automatically an embedding). Then the centralizer of the image of sVec in $\mathcal{C}$ is slightly degenerate.

Let $\mathcal{C}$ be a slightly degenerate braided fusion category. Below we recall some facts about $\mathcal{C}$ from $\mathrm{DNO}, \mathrm{BNRW}$.

Let $\delta$ denote the simple object generating $\mathcal{Z}_{\text {sym }}(\mathcal{C})$. Then $\delta \otimes X \not X$ for each simple object $X$ in $\mathcal{C}$ (see [Mu1, Lemma 5.4] and [DGNO, Lemma 3.28]). In particular, the rank of a slightly degenerate braided fusion category is even.

We say that $\mathcal{C}$ is split if $\mathcal{C} \cong \mathcal{C}_{0} \otimes \mathrm{sVec}$, where $\mathcal{C}_{0}$ is a non-degenerate braided fusion category. Any pointed slightly degenerate braided fusion category is split, see [ENO3, Proposition 2.6(ii)] or [DGNO, Corollary A.19].

The following definition is due to Müger $\mathrm{Mu} 2$.

Definition 2.3. A minimal extension of a slightly degenerate braided fusion (respectively, super-modular) category $\mathcal{C}$ is a braided tensor functor $\iota: \mathcal{C} \hookrightarrow \tilde{\mathcal{C}}$, where $\tilde{\mathcal{C}}$ is a non-degenerate braided fusion (respectively, modular) category such that the centralizer of $\mathcal{C}$ in $\tilde{\mathcal{C}}$ is the image of sVec.

Note that the above functor $\iota$ is an embedding by [DMNO, Corollary 3.26].

Clearly, every slightly degenerate braided fusion category that admits a minimal extension can obtained via the construction from Example 2.2 and vice versa.

An equivalence of minimal extensions is defined in an obvious way.

Example 2.4. The category $\mathrm{sVec}$ has 16 inequivalent minimal extensions DNO, Kt: 8 Ising categories and 8 pointed categories. The Witt classes of these extensions form a subgroup of the categorical Witt group isomorphic to $\mathbb{Z} / 16 \mathbb{Z}$.

It follows that $\mathrm{FPdim}(\tilde{\mathcal{C}})=2 \mathrm{FPdim}(\mathcal{C})$. By $[\mathrm{Mu1}$, DGNO] this is the minimal possible value of the Frobenius-Perron dimension of a non-degenerate braided fusion category containing $\mathcal{C}$. This explains our terminology.

Lemma 2.5. Let $\mathcal{D}$ be a fusion category and let $\mathcal{D}_{0} \subset \mathcal{D}$ be a fusion subcategory such that $F P \operatorname{dim}(\mathcal{D})=2 F P \operatorname{dim}\left(\mathcal{D}_{0}\right)$. Then $\mathcal{D}$ is faithfully $\mathbb{Z} / 2 \mathbb{Z}$-graded with the trivial component $\mathcal{D}_{0}$. 
Proof. Let $\mathcal{D}=\mathcal{D}_{0} \oplus \mathcal{D}_{1}$ be a decomposition of $\mathcal{D}$ into the sum of $\mathcal{D}_{0}$ and its direct complement $\mathcal{D}_{1}$. Then $\mathcal{D}_{1}$ is a $\mathcal{D}_{0}$-bimodule subcategory of $\mathcal{D}$. To prove the statement it suffices to check that the tensor product of $\mathcal{D}$ maps $\mathcal{D}_{1} \times \mathcal{D}_{1}$ to $\mathcal{D}_{0}$. Let $d:=\operatorname{FP} \operatorname{dim}\left(\mathcal{D}_{0}\right)=\operatorname{FPdim}\left(\mathcal{D}_{1}\right)$. Let $R$ denote the (virtual) regular object in $\mathcal{C}$. We can write it as $R=R_{0}+R_{1}$, where $R_{0}$ is the regular object of $\mathcal{D}_{0}$ and $R_{1}$ is a regular object of the $\mathcal{D}_{0}$-module category $\mathcal{D}_{1}$ ENO1 such that $\operatorname{FPdim}\left(R_{0}\right)=\operatorname{FPdim}\left(R_{1}\right)=d$. Then $R_{1} R=d R=d\left(R_{0}+R_{1}\right)$. On the other hand,

$$
R_{1} R=R_{1}\left(R_{0}+R_{1}\right)=R_{1} R_{0}+R_{1}^{2}=d R_{1}+R_{1}^{2},
$$

since a regular object of $\mathcal{D}_{1}$ is unique up to a scalar multiple. Hence, $R_{1}^{2}=d R_{0}$, which implies that the tensor product of any two objects of $\mathcal{D}_{1}$ is in $\mathcal{D}_{0}$.

Thus, a minimal extension of a slightly degenerate braided fusion category is the same thing as a faithful $\mathbb{Z} / 2 \mathbb{Z}$-extension which is a non-degenerate braided fusion category.

\section{Maximal Rank Bimodule CATEgories}

In this section, we show that invertible bimodule categories over a fusion category exhibit a rank bound, and that the bimodule categories realizing this bound actually form a subgroup of the Brauer-Picard group. We refer the reader to [ENO2] for definitions and properties of invertible bimodule categories.

Proposition 3.1. Let $\mathcal{C}, \mathcal{D}$ be fusion categories, and $\mathcal{M}$ an invertible $(\mathcal{C}-\mathcal{D})$ bimodule category. Then $\operatorname{rank}(\mathcal{M}) \leq(\operatorname{rank}(\mathcal{C}) \operatorname{rank}(\mathcal{D}))^{\frac{1}{2}}$. In particular, for an invertible $\mathcal{C}-\mathcal{C}$ bimodule category, $\operatorname{rank}(\mathcal{M}) \leq \operatorname{rank}(\mathcal{C})$.

Proof. First consider $\mathcal{M}$ as a left $\mathcal{C}$-module category. Then the associated full center provides us with a Lagrangian algebra $L \in \mathcal{Z}(\mathcal{C})$ [D2]. Let $F_{\mathcal{C}}: \mathcal{Z}(\mathcal{C}) \rightarrow \mathcal{C}$ be the forgetful functor, and $I_{\mathcal{C}}$ its adjoint. Then as an algebra in $\mathcal{C}, F_{\mathcal{C}}(L) \cong$ $\bigoplus_{M \in \operatorname{Irr}(\mathcal{M})} \underline{\operatorname{Hom}}(M, M)$, where the internal hom is taken as a left $\mathcal{C}$ module category. Note that each $\underline{\operatorname{Hom}}(M, M)$ is a separable, connected algebra, and thus $\operatorname{dim}\left(\operatorname{Hom}_{\mathcal{C}}\left(\mathbb{1}, F_{\mathcal{C}}(L)\right)=\overline{\operatorname{rank}}(\mathcal{M})\right.$. But we have a canonical isomorphism

$$
\operatorname{Hom}_{\mathcal{C}}\left(\mathbb{1}, F_{\mathcal{C}}(L)\right) \cong \operatorname{Hom}_{\mathcal{Z}(\mathcal{C})}\left(I_{\mathcal{C}}(\mathbb{1}), L\right) .
$$

However, by ENO2, the bimodule category $M$ induces a canonical braided equivalence $\alpha: \mathcal{Z}(\mathcal{C}) \rightarrow \mathcal{Z}(\mathcal{D})$ such that $\alpha(L) \cong I_{\mathcal{D}}(\mathbb{1})$, thus we have

$$
\begin{aligned}
\operatorname{dim}\left(\operatorname{End}_{\mathcal{Z}(\mathcal{C})}\left(I_{\mathcal{C}}(\mathbb{1})\right)\right) & =\operatorname{dim}\left(\operatorname{Hom}_{\mathcal{C}}\left(\mathbb{1}, F_{\mathcal{C}}\left(I_{\mathcal{C}}(\mathbb{1})\right)\right)\right)=\operatorname{rank}(\mathcal{C}) \\
\operatorname{dim}\left(\operatorname{End}_{\mathcal{Z}(\mathcal{C})}(L)\right) & =\operatorname{dim}\left(\operatorname{End}_{\mathcal{Z}(\mathcal{D})}\left(I_{\mathcal{D}}(\mathbb{1})\right)\right)=\operatorname{rank}(\mathcal{D}) .
\end{aligned}
$$

Here we have used that as an object $F_{\mathcal{C}}(I(\mathbb{1})) \cong \bigoplus_{X \in \operatorname{Irr}(\mathcal{C})} X \otimes X^{*}$. Therefore by the Cauchy-Schwartz inequality,

$$
\begin{aligned}
& \operatorname{rank}(\mathcal{M})=\operatorname{dim}\left(\operatorname{Hom}_{\mathcal{Z}(\mathcal{C})}(I(\mathbb{1}), L)\right) \\
& =\sum_{X \in \operatorname{Irr}(\mathcal{Z}(\mathcal{C}))} \operatorname{dim}\left(\operatorname{Hom}_{\mathcal{Z}(\mathcal{C})}(I(\mathbb{1}), X)\right) \operatorname{dim}\left(\operatorname{Hom}_{\mathcal{Z}(\mathcal{C})}(L, X)\right) \\
& \leq \operatorname{dim}\left(\operatorname{End}_{\mathcal{Z}(\mathcal{C})}(I(\mathbb{1}))\right)^{\frac{1}{2}} \operatorname{dim}\left(\operatorname{End}_{\mathcal{Z}(\mathcal{C})}(L)\right)^{\frac{1}{2}} \\
& =(\operatorname{rank}(\mathcal{C}) \operatorname{rank}(\mathcal{D}))^{\frac{1}{2}} \text {. }
\end{aligned}
$$


Remark 3.2. Note the bound $\operatorname{rank}(\mathcal{M}) \leq \operatorname{rank}(\mathcal{C})$ requires invertibility. Consider for example the rank 4 fusion category $\mathcal{C}=\operatorname{Rep}\left(D_{5}\right)$, where $D_{5}$ is the group of symmetries of the regular pentagon. Then there exists a rank 5 indecomposable bimodule category, namely $\operatorname{Rep}\left(\mathbb{Z}_{5}\right)$, where the (left and right) actions of $\operatorname{Rep}\left(D_{5}\right)$ are induced from the restriction functor (here $\mathbb{Z}_{5}$ is the subgroup of rotations of $\left.D_{5}\right)$.

The above proposition leads us to the following definition.

Definition 3.3. We say that an invertible $\mathcal{C}$-bimodule category $\mathcal{M}$ has maximal $\operatorname{rank}$ if $\operatorname{rank}(\mathcal{M})=\operatorname{rank}(\mathcal{C})$.

Proposition 3.4. Let $\Psi: \operatorname{BrPic}(\mathcal{C}) \rightarrow \operatorname{Aut}_{b r}(\mathcal{Z}(\mathcal{C}))$ be the canonical group isomorphism of ENO2. Then $\mathcal{M}$ is maximal rank if and only if $\Psi(\mathcal{M})$ preserves the isomorphism class of the object $I(\mathbb{1})$.

Proof. Returning to the proof of Proposition 3.1 and identifying $\mathcal{D}$ with $\mathcal{C}$ then $\Psi(\mathcal{M})=\alpha$, and we are interested in the case when the Cauchy-Schwartz inequality yields equality. But this happens precisely when there exists a scalar $\lambda$ such that

$$
\operatorname{dim}\left(\operatorname{Hom}_{\mathcal{Z}(\mathcal{C})}(I(\mathbb{1}), X)\right)=\lambda \operatorname{dim}\left(\operatorname{Hom}_{\mathcal{Z}(\mathcal{C})}(\alpha(I(\mathbb{1})), X)\right) .
$$

But

$$
\begin{aligned}
\operatorname{rank}(\mathcal{C}) & =\sum_{X \in \operatorname{Irr}(\mathcal{Z}(\mathcal{C})} \operatorname{dim}\left(\operatorname{Hom}_{\mathcal{Z}(\mathcal{C})}(I(\mathbb{1}), X)\right)^{2} \\
& =\lambda^{2} \sum_{X \in \operatorname{Irr}(\mathcal{Z}(\mathcal{C})} \operatorname{dim}\left(\operatorname{Hom}_{\mathcal{Z}(\mathcal{C})}(\alpha(I(\mathbb{1})), X)\right)^{2}=\lambda^{2} \operatorname{rank}(\mathcal{C}) .
\end{aligned}
$$

Since the dimension of morphism spaces is non-negative, we see that we must have $\lambda=1$. Thus

$$
\operatorname{dim}\left(\operatorname{Hom}_{\mathcal{Z}(\mathcal{C})}(I(\mathbb{1}), X)\right)=\operatorname{dim}\left(\operatorname{Hom}_{\mathcal{Z}(\mathcal{C})}(\alpha(I(\mathbb{1})), X)\right)
$$

for all $X \in \operatorname{Irr}(\mathcal{Z}(\mathcal{C}))$ and the conclusion follows.

Corollary 3.5. The maximal rank invertible bimodule categories form a subgroup of $\operatorname{BrPic}(\mathcal{C})$.

This result seems somewhat surprising, since in general the behavior of the rank of bimodule categories is notoriously difficult to understand under relative tensor products.

Recall there is a canonical subgroup $\operatorname{Out}(\mathcal{C}) \leq \operatorname{BrPic}(\mathcal{C})$ which consists of equivalence classes of invertible bimodule categories which are trivial as a left module category. This implies the right action must be the usual right action twisted by an auto-equivalence of $\mathcal{C}$. More explicitly, let $\beta$ be a tensor autoequivalence of $\mathcal{C}$ and $\mathcal{C}_{\beta}$ the associated bimodule category, which is $\mathcal{C}$ as an underlying category and with actions $X \triangleright Y=X \otimes Y, X \triangleleft Y=X \otimes \beta(Y)$, and the obvious associators. The image of these bimodule categories in $\operatorname{BrPic}(\mathcal{C})$ forms the subgroup $\operatorname{Out}(\mathcal{C})$.

Using the correspondence between module categories and Lagrangian algebras, we see that this is precisely the subgroup of $\operatorname{BrPic}(\mathcal{C})$ which preserve $I(\mathbb{1})$ as an algebra object. In particular, $\operatorname{Out}(\mathcal{C})$ forms a subgroup of the maximal rank bimodule categories. In many cases, this is the whole group. 
Proposition 3.6. For any pointed fusion category $\mathcal{C}$, the group of maximal rank bimodule categories is $\operatorname{Out}(\mathcal{C})$.

Proof. Any pointed fusion category $\mathcal{C}$ is monoidally equivalent to $\operatorname{Vec}(G, \omega)$ for a finite group $G$ and 3-cocycle $\omega \in \mathrm{Z}^{3}\left(G, \mathbb{C}^{\times}\right)$. By $\underline{\mathrm{O} 2}$, the module categories for this fusion category are classified by subgroups $H \leq G$ together with a trivialization of $\left.\omega\right|_{H}$. The rank of the resulting module category is the index $[G: H]$. Thus there is a unique rank $|G|$ indecomposable module category, where $H=\{e\}$, which is $\operatorname{Vec}(G, \omega)$ acting on itself. The dual category is thus $\operatorname{Vec}(G, \omega)$, hence any invertible rank $|G|$ bimodule category is of the form $\operatorname{Out}(\mathcal{C})$.

There exist maximal rank invertible bimodule categories that are not of the form Out $(\mathcal{C})$. One such example is constructed by Ostrik in the appendix of [CMS] using an extension of the Izumi-Xu fusion category. See [CMS, Theorem A.5.1] and [03, Remark 2.19 and Example 2.20].

To find a maximal rank bimodule category not of the form $\operatorname{Out}(\mathcal{C})$, we need not only a distinct etale algebra structure on $I(\mathbb{1})$, but we need this algebra structure to be the image of $I(\mathbb{1})$ under a braided autoequivalence, which makes finding invertible bimodule categories not of the form $\operatorname{Out}(\mathcal{C})$ difficult in general.

To find such examples, we move in a different direction. If $\mathcal{C}$ is braided, we can try to understand invertible module categories over $\mathcal{C}$. Recall from DN1, Remark $2.13]$ that we can characterize the bimodule categories $\mathcal{M} \in \operatorname{BrPic}(\mathcal{C})$ which are in the image of the map from $\operatorname{Pic}(\mathcal{C})$ as the one-sided bimodule categories. By definition, these are bimodule categories for which there exists natural isomorphisms $d_{M, X}: M \triangleleft X \cong X \triangleright M$ satisfying a collection of coherences. It is not hard to see that these coherences imply the only one-sided invertible bimodule category which is trivial as a left module category is the trivial bimodule category $\mathcal{C}$. Thus all nontrivial maximal rank invertible module categories are not of the form $\operatorname{Out}(\mathcal{C})$ and thus provide interesting examples.

We will now provide a characterization of maximal rank invertible module categories for non-degenerate fusion categories in terms of braided autoequivalences. In [D1, Davydov introduced the notion of a soft monoidal functor, which is simply a monoidal functor which is isomorphic to the identity functor as a linear functor. Equivalently, a soft monoidal functor is one which fixes equivalence classes of objects.

Recall from [ENO2, DN1, Section 2.9], $\alpha$-induction provides us with an isomorphism $\partial: \operatorname{Pic}(\mathcal{C}) \rightarrow \operatorname{Aut}^{b r}(\mathcal{C})$. The following result is originally due to Kirillov $\mathrm{Jr}$ Kr] (see also [T, Section II.3]) in the case of modular categories.

Proposition 3.7. If $\mathcal{C}$ is a non-degenerate braided fusion category and $\mathcal{M}$ is an invertible module category, the rank of $\mathcal{M}$ is the number of equivalence classes of simple objects fixed by $\partial(\mathcal{M})$. In particular, the image of the group of maximal rank invertible module categories is the group of soft braided tensor autoequivalences of $\mathcal{C}$.

Proof. $\mathcal{M}$ induces a braided autoequivalence of $\Psi(\mathcal{M}) \in \mathcal{Z}(\mathcal{C})$, which by DN1, Lemma 4.4$]$ is $\operatorname{Id}_{\mathcal{C}} \otimes \partial$, acting on $\mathcal{Z}(\mathcal{C}) \cong \mathcal{C} \otimes \mathcal{C}^{\text {rev }}$. But

$$
I(\mathbb{1}) \cong \bigoplus_{X \in \operatorname{Irr}(\mathcal{C})} X \otimes X^{*}
$$


hence

$$
\Psi(\mathcal{M})(I(\mathbb{1}))=\bigoplus_{X \in \operatorname{Irr}(\mathcal{C})} X \otimes \partial(\mathcal{M})\left(X^{*}\right) .
$$

Thus $\operatorname{rank}(\mathcal{M})=\operatorname{dim}\left(\operatorname{Hom}_{\mathcal{C} \otimes \mathcal{C}^{\text {rev }}}(I(\mathbb{1}), \Psi(\mathcal{M})(I(\mathbb{1})))\right)$ is precisely the number of fixed points of $\partial(\mathcal{M})$ acting on $\operatorname{Irr}(\mathcal{C})$.

Davydov [D1] has computed the group of soft braided autoequivalences for the non-degenerate braided tensor category $\mathcal{Z}(\operatorname{Vec}(G))$ for finite groups $G$. The answer is somewhat involved, but he shows it is a certain subgroup of the image of $\operatorname{Out}(\operatorname{Vec}(G)) \cong H^{2}\left(G, \mathbb{C}^{\times}\right) \rtimes \operatorname{Out}(G)$ inside $\operatorname{Aut}^{b r}(\mathcal{Z}(\operatorname{Vec}(G)))$ satisfying a compatibility condition with respect to double class functions [D1, Theorem 2.12. He then presents several examples which have non-trivial soft braided autoequivalences, the smallest of which has order 64, though there may certainly be smaller examples. In any case, these provide examples of non-trivial maximal rank invertible module categories.

\section{RANK FINITENESS FOR BRAIDED FUSION CATEGORIES}

The rank finiteness theorem for modular categories was proved in BNRW]. It states that up to a braided equivalence there exists only finitely many modular categories of any given rank. Below we extend this result to braided fusion categories that are not necessarily spherical or non-degenerate. The plan is first to establish this result for non-degenerate and slightly degenerate categories and then pass to equivariantizations.

Corollary 4.1. Let $\mathcal{C}=\oplus_{a \in A} \mathcal{C}_{a}$ be a fusion category faithfully graded by a group $A$. Then $\operatorname{rank}(\mathcal{C}) \leq|A| \operatorname{rank}\left(\mathcal{C}_{e}\right)$.

Proof. The components $\mathcal{C}_{a}$ are invertible $\mathcal{C}_{e}$-bimodule categories so this is immediate from Proposition 3.1 .

Lemma 4.2. Let $\mathcal{C}$ be a fusion category and let $G$ be a finite group acting on $G$. Then

$$
\frac{1}{|G|} \operatorname{rank}(\mathcal{C}) \leq \operatorname{rank}\left(\mathcal{C}^{G}\right) \leq|G| \operatorname{rank}(\mathcal{C}) .
$$

Proof. Simple objects of $\mathcal{C}^{G}$ are parameterized by pairs consisting of orbits of simple objects of $\mathcal{C}$ under the action of $G$ and certain irreducible projective representations of stabilizers. Each orbit has at most $|G|$ elements, so the number of orbits is at least $\operatorname{rank}(\mathcal{C}) /|G|$. This implies the first inequality.

On the other hand, there are at $\operatorname{most} \operatorname{rank}(\mathcal{C})$ orbits and each stabilizer has at most $|G|$ irreducible projective representations, which gives the second inequality.

Proposition 4.3. There are finitely many equivalence classes of non-degenerate braided fusion categories of any given rank.

Proof. Let $N$ be a positive integer. By [BNRW], it suffices to show that there is a positive integer $M$ such that any non-degenerate braided fusion category $\mathcal{C}$ of rank $N$ is a subquotient of a modular category of rank $\leq M$. Here by a subquotient we mean a surjective image of a subcategory. Let $\tilde{\tilde{\mathcal{C}}}$ be the sphericalization of $\mathcal{C}$ ENO1]. It is a degenerate ribbon category (its symmetric center is $\operatorname{Rep}(\mathbb{Z} / 2 \mathbb{Z}$ ) with a non-unitary ribbon structure) of rank $2 N$. 
As $\tilde{\mathcal{C}}$ is a $\mathbb{Z} / 2 \mathbb{Z}$-equivariantization of $\mathcal{C}$, its center $\mathcal{Z}(\tilde{\mathcal{C}})$ is a $\mathbb{Z} / 2 \mathbb{Z}$-graded modular category with the trivial component $\mathcal{Z}(\tilde{\mathcal{C}})_{0}=\mathcal{Z}(\mathcal{C})^{\mathbb{Z} / 2 \mathbb{Z}}$ by GNN. Using Corollary 4.1 and Lemma 4.2 we estimate

$$
\operatorname{rank}(\mathcal{Z}(\tilde{\mathcal{C}})) \leq 2 \operatorname{rank}\left(\mathcal{Z}(\tilde{\mathcal{C}})_{0}\right)=2 \operatorname{rank}\left(\mathcal{Z}(\mathcal{C})^{\mathbb{Z} / 2 \mathbb{Z}}\right) \leq 4 \operatorname{rank}(\mathcal{Z}(\mathcal{C}))=4 N^{2}
$$

so one can take $M=4 N^{2}$. Indeed, $\mathcal{C}$ is a quotient of $\tilde{\mathcal{C}}$ and so is a subquotient of $\mathcal{Z}(\tilde{\mathcal{C}})$.

Let $\mathcal{C}_{1}, \mathcal{C}_{2}$ be braided fusion categories with embeddings sVec $\hookrightarrow \mathcal{Z}_{\text {sym }}\left(\mathcal{C}_{i}\right), i=$ 1,2 . Then $\mathcal{C}_{1} \otimes_{\mathrm{sVec}} \mathcal{C}_{2}$ has a canonical structure of a braided fusion category [DNO. Namely, it is equivalent to the category of $A$-modules in $\mathcal{B}_{1} \otimes \mathcal{B}_{2}$, where $A$ is the regular algebra of the maximal Tannakian subcategory of $\mathrm{sVec} \otimes \operatorname{sVec} \subset \mathcal{C}_{1} \otimes \mathcal{C}_{2}$. If $\mathcal{C}_{1}$ and $\mathcal{C}_{2}$ are slightly degenerate then so is $\mathcal{C}_{1} \nabla_{\text {sVec }} \mathcal{C}_{2}$.

Proposition 4.4. There are finitely many equivalence classes of slightly degenerate braided fusion categories of any given rank.

Proof. Let $\mathcal{C}$ be a slightly degenerate braided fusion category of rank $N$. Its center $\mathcal{Z}(\mathcal{C})$ contains a fusion subcategory $\mathcal{C} \vee \mathcal{C}^{\text {rev }} \cong \mathcal{C} \nabla_{\mathrm{sVec}} \mathcal{C}^{\text {rev }}$ of FrobeniusPerron dimension $\frac{1}{2} \mathrm{FPdim}(\mathcal{C})^{2}=\frac{1}{2} \mathrm{FPdim}(\mathcal{Z}(\mathcal{C}))$. Hence, $\mathcal{Z}(\mathcal{C})$ is $\mathbb{Z} / 2 \mathbb{Z}$-graded by Lemma 2.5 and

$$
\operatorname{rank}(\mathcal{Z}(\mathcal{C})) \leq 2 \operatorname{rank}\left(\mathcal{C} \nabla_{\mathrm{sVec}} \mathcal{C}^{\text {rev }}\right)=2 \times \frac{N^{2}}{2}=N^{2}
$$

by Corollary 4.1. Since $\mathcal{C}$ is a fusion subcategory of $\mathcal{Z}(\mathcal{C})$ the result follows.

Remark 4.5. It was observed in BGNPRW, following BRWZ that if $\mathcal{C} \subset \tilde{\mathcal{C}}$ is a minimal modular extension of a super-modular category then $\frac{3}{2} \operatorname{rank}(\mathcal{C}) \leq$ $\operatorname{rank}(\tilde{\mathcal{C}}) \leq 2 \operatorname{rank}(\mathcal{C})$. This could be used in place of the more general Corollary 4.1 in the proof above.

Theorem 4.6. There are finitely many equivalence classes of braided fusion categories of any given rank.

Proof. Let $\mathcal{C}$ be a braided fusion category of rank $N$. Let $\mathcal{E} \cong \operatorname{Rep}(G)$ be the maximal Tannakian subcategory of $\mathcal{Z}_{\text {sym }}(\mathcal{C})$. Then $\mathcal{C}=\mathcal{D}^{G}$, where $\mathcal{D}$ is either non-degenerate or slightly degenerate braided fusion category. By Lemma 4.2

$$
\operatorname{rank}(\mathcal{D}) \leq|G| \operatorname{rank}(\mathcal{C})=|G| N .
$$

Now let $M$ be the maximal order of a group with at most $N$ isomorphism classes of irreducible representations ( $M$ exists since the number of such groups is finite by Landau's theorem). We have $\operatorname{rank}(\mathcal{D}) \leq M N$, so there are finitely many choices for $\mathcal{D}$, thanks to Lemmas 4.3 and 4.4 There are also finitely many choices for the group $G$ and for each such a choice there are finitely many different actions of $G$ on $\mathcal{D}$ ENO1. Thus, there are finitely many possible $\mathcal{C}$ 's.

Corollary 4.7. There are finitely many equivalence classes of G-crossed braided fusion categories of any given rank.

Proof. Follows immediately from Theorem 4.6 and Lemma 4.2 since any $G$-crossed braided fusion category is obtained as a de-equivariantization of a braided fusion category [DGNO, Theorem 4.4.]. 


\section{The Center of A SLightly Degenerate BRAided Fusion CATEgory}

Let $\mathcal{C}$ be a slightly degenerate braided fusion category. We have $\mathcal{Z}_{\text {sym }}(\mathcal{C}) \cong \mathrm{sVec}$. Let $\delta$ denote the non-trivial invertible object in $\mathcal{Z}_{\text {sym }}(\mathcal{C})$.

For any $\mathcal{C}$-module category $\mathcal{M}$ let us denote

$$
\mathcal{M}^{s}:=\mathcal{M} \otimes_{\mathrm{sVec}} \mathrm{Vec} .
$$

In particular, $\mathcal{C}^{s}:=\mathcal{C} \otimes_{\mathrm{sVec}} \mathrm{Vec}$ is equivalent to the category of $A$-modules in $\mathcal{C}$, where $A$ is the regular algebra of sVec. We have $\mathcal{M}^{s}=\mathcal{M} \otimes_{\mathcal{C}} \mathcal{C}^{s}$. Note that $\operatorname{rank}\left(\mathcal{C}^{s}\right)=\frac{1}{2} \operatorname{rank}(\mathcal{C})$

Lemma 5.1. $\mathcal{C}^{s}$ is an invertible $\mathcal{C}$-module category of order 2.

Proof. This follows from straightforward equivalences:

$$
\mathcal{C}^{s} \otimes_{\mathcal{C}} \mathcal{C}^{s}=\left(\mathcal{C} \otimes_{\mathrm{sVec}} \mathrm{Vec}\right) \bigotimes_{\mathcal{C}}\left(\mathcal{C} \otimes_{\mathrm{sVec}} \mathrm{Vec}\right) \cong \mathcal{C} \otimes_{\mathrm{sVec}}\left(\operatorname{Vec} \otimes_{\mathrm{sVec}} \mathrm{Vec}\right) \cong \mathcal{C}
$$

where we used the obvious fact $\operatorname{Vec} \bigotimes_{\mathrm{sVec}} \mathrm{Vec} \cong \mathrm{sVec}$.

Lemma 5.2. We have $\mathcal{C}^{s} \otimes_{\mathcal{C}} \mathcal{M} \cong \mathcal{M} \nabla_{\mathcal{C}} \mathcal{C}^{s}$ for any $\mathcal{C}$-module category $\mathcal{M}$.

Proof. Let $B \in \mathcal{C}$ be an algebra such that $\mathcal{M} \cong \mathcal{C}_{B}$. Then $A \otimes B \cong B \otimes A$ as algebras since $A \in \mathcal{Z}_{\text {sym }}(\mathcal{C})$. This yields the statement.

Let $\mathcal{C}_{1}, \mathcal{C}_{2}$ be slightly degenerate braided fusion categories. Let

$$
E \in \mathrm{sVec} \otimes \mathrm{sVec} \subset \mathcal{C}_{1} \otimes \mathcal{C}_{2}
$$

be a canonical étale algebra. Recall that the braided fusion category $\mathcal{C}_{1} \otimes_{\mathrm{sVec}} \mathcal{C}_{2}$ is defined as the category of $E$-modules in $\mathcal{C}_{1} \otimes \mathcal{C}_{2}$. There are obvious embeddings $\mathcal{C}_{1}, \mathcal{C}_{2} \hookrightarrow \mathcal{C}_{1} \otimes_{\mathrm{sVec}} \mathcal{C}_{2}$

Let $\mathcal{M}_{1}$ and $\mathcal{M}_{2}$ be module categories over $\mathcal{C}_{1}$ and $\mathcal{C}_{2}$. Define a $\mathcal{C}_{1} \otimes_{\mathrm{sVec}} \mathcal{C}_{2^{-}}$ module category $\mathcal{M}_{1} \otimes_{\mathrm{sVec}} \mathcal{M}_{2}$ to be the category of $E$-modules in $\mathcal{M}_{1} \otimes \mathcal{M}_{2}$ with the module action given by

$$
X \odot M=X \otimes_{E} M, \quad X \in \mathcal{C}_{1} \otimes_{\mathrm{sVec}} \mathcal{C}_{2}, M \in \mathcal{M}_{1} \otimes_{\mathrm{sVec}} \mathcal{M}_{2}
$$

Let $\mathcal{M}$ be an indecomposable $\mathcal{C}_{1} \otimes_{\mathrm{sVec}} \mathcal{C}_{2}$-module category and let

$$
\mathcal{M}=\bigoplus_{i \in I} \mathcal{M}_{i}, \quad \mathcal{M}=\bigoplus_{j \in J} \mathcal{N}_{j}
$$

be its decompositions into direct sums of indecomposable $\mathcal{C}_{1}$-module categories and $\mathcal{C}_{2}$-module categories, respectively.

Proposition 5.3. There exist indecomposable $\mathcal{C}_{i}$-module categories $\mathcal{L}_{i}, i=1,2$, such that $\mathcal{M} \cong \mathcal{L}_{1} \bigotimes_{s \text { Vec }} \mathcal{L}_{2}$ if and only if $\mathcal{M}_{i} \cap \mathcal{N}_{j}$ is an indecomposable sVecmodule category for some $i \in I$ and $j \in J$.

Proof. One implication is obvious.

Suppose that $\mathcal{M}_{i} \cap \mathcal{N}_{j}$ is an indecomposable sVec-module category. There are two possible cases.

(Case 1) $\mathcal{M}_{i} \cap \mathcal{N}_{j} \cong$ sVec. Let $X \in \mathcal{M}_{i} \cap \mathcal{N}_{j}$ be a simple object. Let $\delta_{i}$ denote the non-trivial invertible object in $\mathcal{C}_{i}, i=1,2$. Then $\delta_{i} \otimes X \nRightarrow X$. Let us view $\mathcal{M}$ as a $\mathcal{C}_{1} \otimes \mathcal{C}_{2}$-module category and compute the internal Hom:

$$
\begin{aligned}
& \underline{\operatorname{Hom}}_{\mathcal{C}_{1} \otimes \mathcal{C}_{2}}(X, X) \\
& \cong \underline{\operatorname{Hom}}_{\mathcal{C}_{1}}(X, X) \otimes \underline{\operatorname{Hom}}_{\mathcal{C}_{2}}(X, X) \oplus \underline{\operatorname{Hom}}_{\mathcal{C}_{1}}\left(X, \delta_{1} \otimes X\right) \otimes \underline{\operatorname{Hom}}_{\mathcal{C}_{2}}\left(\delta_{2} \otimes X, X\right) \\
& \left.\cong\left(\underline{\operatorname{Hom}}_{\mathcal{C}_{1}}(X, X) \otimes \underline{\operatorname{Hom}}_{\mathcal{C}_{2}}(X, X)\right)\right) \otimes E,
\end{aligned}
$$


where $E=\mathbf{1} \otimes \mathbf{1} \oplus \delta_{1} \otimes \delta_{2}$ is the canonical algebra in sVec $\nabla \mathrm{sVec} \subset \mathcal{C}_{1} \otimes \mathcal{C}_{2}$. Therefore, as a $\mathcal{C}_{1} \nabla_{\mathrm{sVec}} \mathcal{C}_{2}$-module category, $\mathcal{M} \cong \mathcal{L}_{1} \nabla_{\mathrm{sVec}} \mathcal{L}_{2}$, where $\mathcal{L}_{i}$ is the category of $\underline{\operatorname{Hom}}_{\mathcal{C}_{i}}(X, X)$-modules in $\mathcal{C}_{i}, i=1,2$.

(Case 2) $\mathcal{M}_{i} \cap \mathcal{N}_{j} \cong$ Vec. In this case the $\mathcal{C}_{1} \nabla_{\text {sVec }} \mathcal{C}_{2}$-module category

$$
\left(\mathcal{C}_{1}^{s} \otimes_{\mathrm{sVec}} \mathcal{C}_{2}\right) \otimes_{\mathcal{C}_{1} \bigotimes_{\mathrm{sVec}} \mathcal{C}_{2}} \mathcal{M}
$$

satisfies the condition of (Case 1) above and, hence, is equivalent to $\mathcal{L}_{1} \otimes_{\mathrm{sVec}} \mathcal{L}_{2}$. Consequently, $\mathcal{M} \cong \mathcal{L}_{1}^{s} \bigotimes_{\text {sVec }} \mathcal{L}_{2}$.

Remark 5.4. The pair of module categories $\mathcal{L}_{1}, \mathcal{L}_{2}$ in Proposition 5.3 is determined up to a simultaneous substitution of $\mathcal{L}_{1}, \mathcal{L}_{2}$ by $\mathcal{L}_{1}^{s}, \mathcal{L}_{2}^{s}$.

Example 5.5. Let $\mathcal{C}_{1}=\mathcal{C}_{2}=\mathrm{sVec}$. Then $\mathcal{C}_{1} \otimes_{\mathrm{sVec}} \mathcal{C}_{2}=\mathrm{sVec}$ and

$$
\begin{aligned}
\mathrm{sVec} & \cong \mathrm{sVec} \bigotimes_{\mathrm{sVec}} \mathrm{sVec} \cong \mathrm{Vec} \bigotimes_{\mathrm{sVec}} \mathrm{Vec}, \\
\mathrm{Vec} & \cong \mathrm{Vec} \bigotimes_{\mathrm{sVec}} \mathrm{sVec} \cong \mathrm{sVec} \bigotimes_{\mathrm{sVec}} \mathrm{Vec}
\end{aligned}
$$

as sVec-module categories.

Proposition 5.6. Let $\mathcal{C}$ be a slightly degenerate braided fusion category and let $\mathcal{D}=$ $\mathcal{D}_{0} \oplus \mathcal{D}_{1}$ be a minimal extension (see Definition 2.3) of $\mathcal{D}_{0}:=\mathcal{C} \nabla_{\text {svec }} \mathcal{C}^{\text {rev }}$. There exists an invertible $\mathcal{C}$-module (respectively, $\mathcal{C}^{\text {rev }}$-module) category $\mathcal{M}$ (respectively, $\mathcal{N}$ ) such that $\mathcal{D}_{1} \cong \mathcal{M} \nabla_{\text {sVec }} \mathcal{N}$ as a $\mathcal{C} \nabla_{\text {sVec }} \mathcal{C}^{\text {rev }}$-module category.

The equivalence classes of module categories $\mathcal{M}$ and $\mathcal{N}$ are determined up to a simultaneous substitution by $\mathcal{M}^{s}$ and $\mathcal{N}^{s}$.

Proof. Note that $\mathcal{D}$ is a $\mathbb{Z} / 2 \mathbb{Z}$-graded extension of $\mathcal{D}_{0}$ by Lemma 2.5.

Let $n$ be the number of $\mathcal{C}$-module components of $\mathcal{D}_{1}$. By [DGNO, Corollary 3.6] the number of $\mathcal{C}$-module components of $\mathcal{D}$ is equal to the rank of the centralizer of $\mathcal{C}$ in $\mathcal{D}$. The latter is $\mathcal{C}^{\text {rev }}$. Since the number of $\mathcal{C}$-module components in $\mathcal{D}_{0}=$ $\mathcal{C} \otimes_{\text {sVec }} \mathcal{C}^{\text {rev }}$ is $\frac{1}{2} \operatorname{rank}(\mathcal{C})$ we conclude that

$$
n=\frac{1}{2} \operatorname{rank}(\mathcal{C})
$$

Note that $n$ is also equal to the number of $\mathcal{C}^{\text {rev }}$-module components of $\mathcal{D}_{1}$.

Let $\oplus_{i=1}^{n} \mathcal{M}_{i}$ (respectively, $\oplus_{j=1}^{n} \mathcal{N}_{j}$ ) be decompositions of $\mathcal{D}_{1}$ into direct sums of indecomposable $\mathcal{C}$-module (respectively, $\mathcal{C}^{\text {rev }}$-module) subcategories. In view of Proposition 5.3 it suffices to check that for some $i, j$ the intersection $\mathcal{M}_{i} \cap \mathcal{N}_{j}$ is an indecomposable sVec-module category.

By Proposition 3.1 we have

$$
\operatorname{rank}\left(\mathcal{D}_{1}\right) \leq \operatorname{rank}\left(\mathcal{D}_{0}\right)=\frac{1}{2} \operatorname{rank}(\mathcal{C})^{2}=2 n^{2}
$$

Since $\mathcal{D}_{1}$ is indecomposable as a $\mathcal{D}_{0}$-bimodule category each $\mathcal{M}_{i} \cap \mathcal{N}_{j}, i, j=1, \ldots, n$ is non-zero. If any of these intersections has rank 1 , then it is sVec-indecomposable. This happens automatically if either $\operatorname{rank}\left(\mathcal{M}_{i}\right)$ or $\operatorname{rank}\left(\mathcal{N}_{j}\right)$ is less than $2 n$ for some $i$ or $j$ (indeed, $\operatorname{Irr}\left(\mathcal{M}_{i}\right)$ intersects non-trivially with $n$ disjoint $\operatorname{sets} \operatorname{Irr}\left(\mathcal{N}_{j}\right), j=$ $1, \ldots, n)$.

So let us assume that all intersections $\mathcal{M}_{i} \cap \mathcal{N}_{j}$ have rank $\geq 2$ and that all $\mathcal{M}_{i}$ and $\mathcal{N}_{j}$ have rank $\geq 2 n$. The latter implies that $\operatorname{rank}\left(\mathcal{M}_{i}\right)=\operatorname{rank}\left(\mathcal{N}_{j}\right)=2 n$ and $\operatorname{rank}\left(\mathcal{M}_{i} \cap \mathcal{N}_{j}\right)=2$ for all $i$ and $j$ since otherwise $\operatorname{rank}\left(\mathcal{D}_{1}\right)>2 n \times n=2 n^{2}$. Hence, $\operatorname{rank}\left(\mathcal{D}_{1}\right)=2 n^{2}=\operatorname{rank}\left(\mathcal{D}_{0}\right)$, i.e., $\mathcal{D}_{1}$ is a maximal rank invertible $\mathcal{D}_{1}$-bimodule category. 
By Proposition 3.4 the Lagrangian algebras corresponding to $\mathcal{D}_{0}$-bimodule categories $\mathcal{D}_{0}$ and $\mathcal{D}_{1}$ are isomorphic as objects of $\mathcal{Z}\left(\mathcal{D}_{0}\right)$. In particular, their forgetful images in $\mathcal{D}_{0}$ are isomorphic:

$$
\bigoplus_{X \in \operatorname{Irr}\left(\mathcal{D}_{0}\right)} X \otimes X^{*} \cong \bigoplus_{X \in \operatorname{Irr}\left(\mathcal{D}_{1}\right)} X \otimes X^{*}
$$

The object on the left does not contain $\delta$ since $\delta$ acts freely on $\operatorname{Irr}\left(\mathcal{D}_{0}\right)$ by DGNO, Lemma 3.28]. Hence, the same is true for the object on the right, i.e., $\delta$ also acts freely on $\operatorname{Irr}\left(\mathcal{D}_{1}\right)$. Thus, every $\mathcal{M}_{i} \cap \mathcal{N}_{j}$ is sVec-indecomposable and $\mathcal{D}_{1}=\mathcal{M} \bigotimes_{\text {sVec }} \mathcal{N}$ by Proposition 5.3

The following equivalences:

$$
\begin{aligned}
& \mathcal{D}_{0} \cong \mathcal{D}_{1} \otimes_{\mathcal{D}_{0}} \mathcal{D}_{1}
\end{aligned}
$$

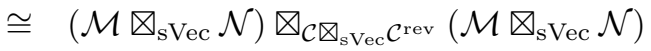

$$
\begin{aligned}
& \cong\left(\mathcal{M} \nabla_{\mathcal{C}} \mathcal{M}\right) \nabla_{\mathrm{sVec}}\left(\mathcal{N} \nabla_{\mathcal{C}^{\mathrm{rev}}} \mathcal{N}\right) \text {, }
\end{aligned}
$$

imply that $\mathcal{M} \otimes_{\mathcal{C}} \mathcal{M}$ is equivalent to $\mathcal{C}$ or $\mathcal{C}^{s}$ and, hence, $\mathcal{M}$ is invertible. Similarly, $\mathcal{N}$ is invertible.

Corollary 5.7. Let $\mathcal{C}$ be a slightly degenerate braided fusion category. There exist an invertible $\mathcal{C}$-module categories $\mathcal{M}$ and $\mathcal{N}$ such that

$$
\mathcal{Z}(\mathcal{C}) \cong\left(\mathcal{C} \nabla_{s \text { Vec }} \mathcal{C}^{\text {rev }}\right) \oplus\left(\mathcal{M} \otimes_{s \text { Vec }} \mathcal{N}\right)
$$

as a $\mathcal{C} \bigotimes_{s V e c} \mathcal{C}^{r e v}$-module category.

Remark 5.8. It is possible to show that the above $\mathcal{M}$ and $\mathcal{N}$ are braided $\mathcal{C}$-module categories of order 2, see DN2].

Remark 5.9. It will be interesting to see if $\mathcal{C}$ is a slightly degenerate braided fusion category then for such an $\mathcal{M}$ as above $\mathcal{C} \oplus \mathcal{M}$ has a structure of a minimal extension of $\mathcal{C}$. One expects that there are 16 choices of $\mathcal{M}$ in this case, by the results of [BGNPRW, KLW]. Notice that if $\tilde{\mathcal{C}}=\mathcal{C} \oplus \mathcal{N}$ is a minimal extension of some slightly degenerate braided fusion category $\mathcal{C}$ then $\mathcal{Z}(\mathcal{C})$ has the form as in Corollary 5.7, as can be seen as follows: $\mathcal{Z}(\tilde{\mathcal{C}}) \cong \tilde{\mathcal{C}} \otimes \tilde{\mathcal{C}}^{\text {rev }}$ contains a Tannakian subcategory $\mathcal{D} \cong \operatorname{Rep}(\mathbb{Z} / 2 \mathbb{Z})$ as the diagonal of sVec $\otimes$ sVec. The centralizer of $\mathcal{D}$ in $\mathcal{Z}(\tilde{\mathcal{C}})$ is $\left(\mathcal{C} \otimes \mathcal{C}^{\text {rev }}\right) \oplus\left(\mathcal{N} \otimes \mathcal{N}^{\text {rev }}\right)$, so that the de-equivariantization is

$$
\left(\mathcal{C} \nabla_{\mathrm{sVec}} \mathcal{C}^{\mathrm{rev}}\right) \oplus\left(\mathcal{N} \nabla_{\mathrm{sVec}} \mathcal{N}^{\mathrm{rev}}\right) \cong\left[\mathcal{Z}(\tilde{\mathcal{C}})_{\mathbb{Z} / 2 \mathbb{Z}}\right]_{0} \cong \mathcal{Z}(\mathcal{C})
$$

\section{REFERENCES}

[BRWZ] P. Bonderson, E. Rowell, Z. Wang, Q. Zhang, Congruence subgroups and super-modular categories, Pacific J. Math. 296 (2), 257-270.

[BGNPRW] P. Bruillard, C. Galindo, S.-H. Ng, J. Plavnik, E. Rowell, and Z. Wang, Classification of super-modular categories by rank, preprint. arXiv:1705.05293

[BNRW] P. Bruillard, S.-H. Ng, E. Rowell, and Z. Wang. Rank-finiteness for modular categories, J. Amer. Math. Soc. 29 (2016), 857-881.

[CMS] F. Calegari, S. Morrison, N. Snyder Cyclotomic integers, fusion categories, and subfactors (with an appendix by V. Ostrik), Comm. Math. Phys., 303 (2011), no. 3, 845-896.

[D1] A. Davydov. Bogomolov multiplier, double class-preserving automorphisms, and modular invariants for orbifolds, J. Math. Phys. 55 (2014), no. 9.

[D2] A. Davydov. Centre of an algebra, Adv. Math. 225, (2010), no. 1, 319-348. 
[DMNO] A. Davydov, M. Müger, D. Nikshych, and V. Ostrik, The Witt group of non-degenerate braided fusion categories, Journal für die reine und angewandte Mathematik, 677 (2013), 135-177.

[DN1] A. Davydov and D. Nikshych. The Picard crossed module of a braided tensor category, Algebra and Number Theory, 3 (2013), no. 6, 1365-1403.

[DN2] A. Davydov and D. Nikshych. Braided module categories and braided extensions, preprint.

[DNO] A. Davydov, D. Nikshych, and V. Ostrik. On the structure of the Witt group of nondegenerate braided fusion categories, Selecta Mathematica 19 (2013), no. 1, 237-269.

[DGNO] V. Drinfeld, S. Gelaki, D. Nikshych, and V. Ostrik. On braided fusion categories I, Selecta Mathematica, 16 (2010), no. 1, 1-119.

[EGNO] P. Etingof, S. Gelaki, D. Nikshych, and V. Ostrik. Tensor categories, Mathematical Surveys and Monographs, 205, American Mathematical Society (2015).

[ENO1] P. Etingof, D. Nikshych, and V. Ostrik. On fusion categories, Annals Math. 162 (2005), 581-642.

[ENO2] P. Etingof, D. Nikshych, and V. Ostrik. Fusion categories and homotopy theory, Quantum Topology, 1 (2010), no. 3, 209-273.

[ENO3] P. Etingof, D. Nikshych, and V. Ostrik. Weakly group-theoretical and solvable fusion categories, Adv. Math. 226 (2011), 176-205.

[GNN] S. Gelaki, D. Naidu, and D. Nikshych, Centers of graded fusion categories, Algebra and Number Theory, 3 (2009), no. 8, 959-990.

[Kr] A. Kirillov Jr, On G-equivariant modular categories, preprint. arXiv:math/0401119

[Kt] A. Kitaev, Anyons in an exactly solved model and beyond, Ann. Physics, 321 (2006) no. 1, $2-111$.

[KLW] T. Lan, L. Kong, X.-G. Wen, Modular extensions of unitary braided fusion categories and 2+1D topological/SPT orders with symmetries. Comm. Math. Phys. 351 (2017), no. 2, 709-739.

[Mu1] M. Müger, Galois theory for braided tensor categories and the modular closure, Adv. Math. 150 (2000), no. 2, 151-201.

[Mu2] M. Müger, Conformal field theory and Doplicher-Roberts reconstruction, Mathematical Physics in Mathematics and Physics 30 (2001): 295.

[O1] V. Ostrik, Fusion categories of rank 2, Math. Res. Lett. 10 (2003), no. 2-3, 177183.

[O2] V. Ostrik, Module categories, weak Hopf algebras and modular invariants, Transform. Group, 8 (2003), no. 2, 177-206.

[O3] V. Ostrik, Pivotal fusion categories of rank 3, Mosc. Math. J., 15 (2015), no. 2, 373-396.

$[\mathrm{T}]$ V. Turaev, Homotopy quantum field theory. Appendix 5 by Michael Müger and Appendices 6 and 7 by Alexis Virelizier. EMS Tracts in Mathematics, 10. European Mathematical Society (EMS), Zürich, 2010. 